\title{
How safe is your DNA extract?
}

\author{
Lester M. Shulman $^{1,3 *}$, Dan David ${ }^{2}$, Ella Mendelson ${ }^{1,3}$ and Daniel Elad ${ }^{2}$ \\ ${ }^{1}$ Central Virology Laboratory, Public Health Services, Israel Ministry of Health, Chaim Sheba Medical Center, Tel Hashomer, Israel \\ ${ }^{2}$ Kimron Veterinary Institute, Beit Dagan, Israel \\ ${ }^{3}$ Department of Epidemiology and Preventive Medicine, School of Public Health, Sackler Faculty of Medicine, Tel Aviv University, Ramat Aviv, Israel
}

${ }^{*}$ Corresponding author: Lester M. Shulman, Central Virology Laboratory, Public Health Services, Israel Ministry of Health, Chaim Sheba Medical Center, Tel Hashomer, Israel; Email: lester.shulman@sheba.health.gov.il

Received: April 14, 2019; Accepted: April 20, 2019; Published: May 16, 2019;

\begin{abstract}
Three of four extraction methods yielding high quality DNA from blood failed to remove all live Bacillus anthracis from the extraction arm of a molecular assay that provided a partial molecular fingerprint of endemic B. anthracis in Israel and distinguished B. anthracis from closely related gram-positive bacteria.
\end{abstract}

Key words: Bacillus anthracis; DNA extraction; biosafety, sequence analysis

\section{Introduction}

Anthrax is an infectious disease caused by the non-motile, grampositive, spore forming bacterium, Bacillus anthracis. Three forms of infection occur depending on the route of infection; cutaneous (skin), inhalation (lungs) and gastrointestinal. Edema toxin, lethal toxin, protective antigen, and capsular antigen are the virulence factors associated with $B$. anthracis pathogenesis. These factors are encoded on two plasmids, pX01 [1] and pX02 [2, 3]. The pX01 plasmid (185 $\mathrm{kb}$ ) encodes the protective antigen, pag [4], the lethal factor, lef [5], and the edema factor, $c y c$ [6], while the pX02 plasmid ( $95 \mathrm{~kb}$ ) encodes three genes required for capsule formation, Cap A, Cap B, and Cap C [7]. Both plasmids must be present for B. anthracis to be pathogenic. Non-pathogenic, live $B$. anthracis agricultural vaccines have been produced from $B$. anthracis strains that lack either the plasmid pX01 (Pasteur vaccine strains) or pX02 (Sterne vaccine strains).

B. anthracis produces very stable spores when growth conditions become less than optimal. These spores remain viable in the soil for years and can infect domestic and wild animal. Humans can become infected with anthrax accidentally after coming in contact with the spores, by handling products from infected animals, by inhaling anthrax spores from contaminated animal products, and by eating undercooked meat from infected animals. Exposure can also be deliberate by acts of war or bioterrorism.

In any suspected anthrax outbreak (infection of one or more organism in an anthrax free region) it is important to know within a clinically relevant time whether pathogenic $B$. anthracis is actually present and in which organisms. A rapid molecular identification technique involves extracting DNA and characterizing it after PCR amplification using published $B$. anthracis specific primers validated for natural and weaponized anthrax and using commercially available extraction systems. The first requirement when establishing such an identification protocol is to determine whether the extracted DNA needs to be treated as a potential biological hazard (e.g., still contained infectious bacteria or spores) or just as a biochemical hazard (e.g., non-infectious DNA that might produce a false positive if reaction mixtures became contaminated).

\section{Methods}

\section{Extraction of DNA from clinical samples spiked with $B$. anthracis}

Bacteremia was mimicked by spiking fresh human blood from blood count Vacutainer (Becton, Dickinson and Company, USA) tubes with bacteria from overnight liquid broth cultures of seven Israeli veterinary bovine isolates of $B$. anthracis isolated between 1980 and1990, obtained from the Clinical Bacteriology Laboratory, The Kimron Veterinary Institute, Israel, from seven non-B. anthracis, gram-positive clinical bacterial isolates obtained from the Bacteriology Laboratory, Sheba Medical Center, Tel Hashomer, Israel, and from Pasteur and Stern B. anthracis vaccine strains. PCR-quality DNA was prepared using four different procedures: DNA extraction using GeneReleaser (Bio Ventures, Incorporated, Murfreesboro, TN, USA), High Pure DNA Extraction Kits (Roche Diagnostics, Mannheim, Germany), and DNA Easy Tissue Kits (QIAGEN GMBH, Hilden, Germany), or by pre-heating aliquots of spiked blood at $95^{\circ} \mathrm{C}$ for 15 minutes before adding the PCR reaction mix.

\section{Biosefety of DNA extracts}

Aliquots of DNA from each procedure were shaken overnight in broth at $37 \mathrm{C}$ to determine whether they still contained any viable $B$. anthracis. 


\section{Molecular identification of $B$. anthracis genomic and plasmid DNA}

The genomic and plasmid primers used in this study for PCR amplification, listed in Table 1, were chosen for the reasons outlined below.

Table 1. PCR primers used to amplify Bacillis anthracis genomic and plasmid DNAs.

\begin{tabular}{|c|c|}
\hline Primer name & Primer sequence \\
\hline \multicolumn{2}{|c|}{ Genomic: vrrA [Ref $(8,9)]$} \\
\hline GPR1 & 5'-CGT AGT TCA CGA ACT GCA TCT-3' \\
\hline GPR2 & 5'-ATG ATG TAT CTA ATG CGG CGT-3' \\
\hline EWA1 & 5'-TAT GGT TGG TAT TGC TG-3' \\
\hline EWA2 & 5'-ATG GTT CCG CCT TAT CG-3' \\
\hline GPR4 & 5'-ACA ACT ACC ACC GAT GGC-3' \\
\hline GPR5 & 5'-TTA TTT ATC ATA TTA GTT GGA TTC g-3' \\
\hline \multicolumn{2}{|c|}{ Genomic: BA813 [Ref $(11,15,14)]$} \\
\hline Ba813 R1 & 5'-TTA ATT CAC TTG CAA CTG ATG GG-3' \\
\hline $\mathrm{Ba} 813 \mathrm{R} 2$ & 5'-AAC GAT AGC TCC TAC ATT TGG AG-3' \\
\hline \multicolumn{2}{|c|}{ Plasmid X01: pag $[\operatorname{Ref}(11,15,14)]$} \\
\hline pag67 & 5'-CAG AAT CAA GTT CCC AGG GG-3' \\
\hline pag68 & 5-'TCG GAT AAG CTG CCA CAA GG-3' \\
\hline Pag23 & 5'-CTA CAG GGG ATT TAT CTA TTC C-3' \\
\hline Pag24 & 5'-ATT GTT ACA TGA TTA TCA GCG G-3' \\
\hline \multicolumn{2}{|c|}{ Plasmid X02: Cap A [Ref (8)] } \\
\hline CapA-F & 5'-CAG AAG CAG TAG CAC CAG TAA-3' \\
\hline CapA-R & 5'-ATT TTC ACC AGC ACC CAC-3' \\
\hline CapA-Fnes & 5'-TGA CGA TGG TTG GTG ACA-3' \\
\hline CapA-Rnes & 5'-CCT TAT TGT ATC TTT AGT TCC C-3' \\
\hline
\end{tabular}

\section{B. anthracis Genomic DNA}

The 1110 nt vrrA template defined by primer pair GPR1 / GPR2 was chosen for the genomic template since it was reported to contain two to six copies of a variable number tandem repeat (VNTR) of 5'caatatcaacaa-3' and primers recognizing this template had been shown to distinguished $B$. anthracis from closely related gram positive bacteria such as Bacillus cereus, B. thuringiensis and B. mycoides [8, 9]. A further advantage is that since the copy number is conserved in progeny [9], the VNTR vrrA copy number would provide a partial $B$. anthracis fingerprint. A full molecular fingerprint of any $B$. anthracis isolate would require a series of PCR reactions targeting this vrrA template and 5 additional genomic and 2 plasmid VNTR sites [10]. While these additional reactions might help distinguish endemic strains from introduced strains, they are not necessary for rapid primary identification of $B$. anthracis infections. Two internal primer pairs were chosen. Depending on VNTR copy number, the GPR4 / GPR5 primer pair amplifies a 378 to $426 \mathrm{nt}$ sub-fragment of vrrA, while the EWA1 / EWA2 pair amplifies a 142 to $190 \mathrm{nt}$ sub-fragment within the GPR4 / GPR5 template. The advantage of using the GPR4/ GPR5 primer pair stems from the fact that it had been validated for weaponized anthrax in an outbreak in the USSR [8], whereas it is easier to distinguish VNTR copy number by gel electrophoresis with the shorter EWA1 / EWA2 pair. Results were compared with the BA813R1 BA3R2 primer pair that amplified another genomic template BA813.

\section{B. anthracis Plasmid DNA}

One genomic template from each plasmid was chosen since pathogenicity required the presence of both plasmids. Specifically, pag and Cap A were chosen to represent the pX01 and pX02 plasmids, respectively, from among published PCR and nested PCR procedures for identifying pag, lef, cyc, and Cap A genes [11-14] since the primers for pag had been validated for many diverse strains including suspected weapon-modified organisms and a large database of sequence information existed for comparative molecular epidemiology of both $[13,7]$.

\section{Preparation of positive control DNA for PCR}

PCR amplification products from genomic DNA, Cap A, and pag from a field isolate of $B$. anthracis amplified using GPR-F / GPR-R, CAP-R / CAP F, and PAG67 / PAG 68 primer pairs, respectively, were cloned in pGEM-T-easy plasmids (Promega, Madison WI, USA) and transfected into JM109 competent bacteria (Promega, Madison WI, USA) according to manufacturers instructions. Plasmid DNA purified using Wizard Plus SV Minipreps DNA Purification System. (Promega, Madison, WI) and overnight cultures of transfected bacteria served as positive controls for all PCR reactions. The expected sizes were 377$425 \mathrm{nt}, 397 \mathrm{nt}$, and $747 \mathrm{nt}$, respectively.

\section{PCR Amplification}

Two different PCR reactions were chosen, one based on a single tube Ready-to-go PCR bead assay (GE Healthcare Amersham Biosciences, Piscataway, NJ, USA) where all reagents except primers are stored at room temperature and the other using a commercial combination of Taq polymerases, in this study AmpliTaq Gold (Applied Biosystems by Life Technologies, Foster City, CA, USA), and optimized five-fold concentrated Taq reaction buffer chosen from among buffers A to $\mathrm{H}$ from a PCR Optimizer Kit (Invitrogen Ltd, Paisley, UK). The optimal buffers for PCR for genomic DNA were buffers $\mathrm{E}$ and to a lesser extent $\mathrm{B}$ for primer pair EWA1 / EWA2, buffer $\mathrm{B}$ for pag primers, and buffers A and B for Cap A primers (12 - 25 pmol of each primer per reaction mix). To simplify and unify procedures, all further amplifications with AmpliTaq Gold were with 5x B buffer (300 $\mathrm{mM}$ Tris- $\mathrm{HCl}, 75 \mathrm{mM}$ ammonium sulfate, and $10 \mathrm{mM}$ magnesium chloride at $\mathrm{pH}$ 8.5). The following amplification conditions were used for PCR: Activation at $93^{\circ} \mathrm{C}$ for $10 \mathrm{~min} ; 60^{\circ}$ for $2 \mathrm{~min} ; 72^{\circ}$ for $2 \mathrm{~min}$; 35 cycles of $93^{\circ} \mathrm{C}$ for 45 seconds, $55^{\circ} \mathrm{C}$ for 45 seconds, and $72^{\circ} \mathrm{C}$ for 90 seconds; and a final elongation at $72^{\circ} \mathrm{C}$ for 10 minutes. PCR products were visualized by ethidium bromide staining after gel electrophoresis on $2 \%$ agarose gels.

\section{DNA sequencing}

The consensus sequences for pag and Cap A amplification products of 701 and 359 nt, respectively, were determined for 
templates amplified with external primer pairs. PCR products were purified after gel electrophoresis using QIAgen MiniElute PCR product kits (QIAgen GMBH, Hilden, Germany), and sequenced on an automatic ABI sequencer (Applied Biosystems Inc., Foster City, CA) by the Biological Services Department of the Weizmann Institute of Science, Rehovot, Israel. The Cap A, pag and vrrA sequences from two isolates have been deposited in the GenBank (accession numbers HQ536626 to HQ53631.

\section{Results}

\section{Biosafety of DNA preparations}

Aliquots of DNA were incubated to determine whether the biohazardous mixture of blood and B. anthracis had been converted into a non-viable biochemical by each of four DNA extraction procedures. Aliquots of DNA were incubated overnight in broth. No viable bacteria were recovered from DNA solutions extracted with the QIAgen DNA Easy Tissue Kit when manufacturers' instructions were followed. In contrast, viable $B$. anthracis was recovered in overnight cultures of DNA prepared from B. anthracis-spiked blood cultures using GeneReleaser (Bio Ventures, Incorporated, Murfreesboro, TN, USA) and High Pure DNA Extraction Kit (Roche Diagnostics, Mannheim, Germany) according to manufacturers' recommendations or after incubation at $95^{\circ} \mathrm{C}$ for 15 minutes. To further reduce the chance for viable bacteria remaining in extracted DNA and to increase DNA yield from gram-positive bacteria, we used the QAIgen DNA Easy Tissue Kit for all further preparations and added a manufacturersuggested option of a 30-minute pre-digestion with $20 \mathrm{mg} / \mathrm{ml}$ lysozyme (Sigma) as a mandatory part of the DNA preparation protocol.

All four DNA preparation procedures yielded PCR quality DNA that was amplifiable by all of the primer sets described in Table 1 in both PCR assays. BA813 genomic primer pairs were able to detect as few as 15 to 40 colony-forming units, whereas vrrA, pag (PAG67/ PAG68) and Cap A (EWA1/EWA2) primer pairs required ten-fold more bacteria in both PCR systems. In non-nested single reaction AmpliTaq Gold PCR, internal primer pairs were much better than external pairs when intensities of amplification products were compared. There was a lower threshold of detection when nested PCR was used for both assays, however in the Ready-to-go assay, a single PCR using internal primers gave bands only slightly less intense than those for nested PCR.

PCR of DNA from all blood samples spiked with Israeli field isolates of $B$. anthracis yielded bands of the expected sizes for vrrA, pag and Cap $A$ for each pair of template specific primers. Those spiked with vaccine strains yielded vrrA and only the appropriate plasmid-encoded genes. Specifically pag template was absent for Pasteur vaccine and Cap A template was absent for Stern vaccine. The consensus sequences for pag, Cap A, and genomic DNA amplification products of 701nt, 348 $\mathrm{nt}$, and $127 \mathrm{nt}$ respectively, from seven Israeli veterinary $B$. anthracis strains isolated between 1980 and 1990 were determined for templates amplified with external primer pairs for pag and Cap A, and internal primers WA1 and WA2 for genomic DNA. All seven Israeli isolates had identical pag sequences, except for nucleotide 50 that was either a $\mathrm{C}$ or a T. All seven isolates had identical Cap A sequences. Finally, there were four perfect repeats of a 5'CAATATCAACAA-3' VNTR in the vrrA genomic sequence as determined by electrophoresis of GPR4 / GPR5 PCR products on 2\% agarose gels and by sequencing. The four perfect repeats were flanked by imperfect repeat elements 5'- CAATATCAACAg-3' and 5'-CAATAcCcgCAA-3' upstream and downstream of the 4 perfect repeats, i.e. the sequence was 5'- CAATATCAACAg_CAATATCAACAA CAATATCAACAA CAATATCAACAA CAATATCAACAA CAATA $\underline{c}$ gaCAA-3' Sequences for all three regions from isolates representing the two variants of pag are available from the GenBank nucleotide sequence database HQ536626 to HQ536630.

\section{Discussion}

We have described conditions for extraction of DNA for Bacillus anthracis diagnosis that can be performed in level 2 national clinical and veterinary laboratories using easily acquired commercial kits and components that can be easily transported to level 1 hospital or field hospitals in an emergency. All four DNA preparation procedures produced PCR quality DNA from spiked blood samples designed to mimic $B$. anthracis bacteremia. Both sets of $B$. anthracis genomic primers amplified the correct template in DNA from all Israeli $B$. anthracis isolates.

Diagnostic results should be provided in a clinical relevant time within the framework of practical biosafety procedures. Biosafety is always an issue when using a procedure to convert a biohazardous biological into a non-biohazardous biochemical. Preparation of PCR-quality B. anthracis DNA is no exception. Three of the four DNA preparation procedures evaluated left viable $B$. anthracis in the DNA solution. The addition of a pre-extraction lysozyme digestion step to further insure destruction of viable bacteria adds only 45 to 60 minutes to a PCR diagnostic procedure that can be completed within five and a half hours.

All of the four DNA extraction procedures may be used, provided that appropriate levels of personal protective equipment and environmental protective measures suitable for potential biohazards from viable $B$. anthracis are used at all times. Equipment must be decontaminated immediately after use and all biological and biochemical material must be disposed under strict isolation and decontamination procedures in less than 24 hours to prevent spore formation by any bacteria that remained viable. DNA solutions may be stored frozen, but unless specifically tested must be considered as biohazardous even when a given procedure has been repeatedly proven safe in the past. This is best illustrated by a recent PubMed notification (X-Promed-Id: 20090331.1226) from March 31, 2009 7: 22: 47 AM IDT, entitled ANTHRAX, LABORATORY EXPOSURE - FRANCE (02) that described exposure from an inadequately heat inactivated sample where "As before, a check loopfull was plated out on sheep agar for each supernatant, but because of the many hundreds of times this had been done before without anything growing [the culture had always been killed], the technician took the 6 vials of heated supernatant out of the Level $3+$ lab and went to the Level 2 DNA laboratory before she had read the check plates the next day..." which in this instance were positive. Testing aliquots for viable $B$. anthracis delays results by a day and precludes moving the assay to 
level 1 laboratory. When overnight broth cultures are positive, the amplified stock of $B$. anthracis must be safely disposed.

In conclusion, don't assume that your DNA extract is free from infectious pathogens; test it routinely to be sure.

\section{Acknowledgement}

The Israel Ministries of Health and Agriculture supported this work. Special thanks for the support of the late Dr. Avraham Mates who headed the Israeli Public Health Services Laboratories.

Conflicts of Interest: The authors affirm that there are no conflicts of interest.

\section{References}

1. Mikesell P, BE Ivins, J D Ristroph, and T M Dreier (1983) Evidence for plasmidmediated toxin production in Bacillus anthracis. Infect Immun 39: 371-6.

2. Green BD, L Battisti, TM Koehler, CB Thorne and BE Ivins (1985) Demonstration of a capsule plasmid in Bacillus anthracis. Infect Immun 49: 291-7.

3. Uchida I, T Sekizaki, K Hashimoto, and N Terakado (1985) Association of the encapsulation of Bacillus anthracis with a 60 megadalton plasmid. J Gen Microbiol 131: 363-7.

4. Vodkin M H and SH Leppla (1983) Cloning of the protective antigen gene of Bacillus anthracis. Cell 34: 693-7.

5. Robertson DL and SH Leppla (1986) Molecular cloning and expression in Escherichia coli of the lethal factor gene of Bacillus anthracis. Gene 44: 71-8.

6. Mock M, E Labruyere, P Glaser, A Danchin, and A Ullmann (1988) Cloning and expression of the calmodulin-sensitive Bacillus anthracis adenylate cyclase in Escherichia coli.Gene 64: 277-84

7. Makino S, C Sasakawa, I Uchida, N Terakado, and M Yoshikawa (1988) Cloning and $\mathrm{CO} 2$-dependent expression of the genetic region for encapsulation from Bacillus anthracis. Mol Microbiol 2: 371-6.

8. Jackson PJ, ME Hugh-Jones, DM Adair, G Green, KK Hill et al (1998) PCR analysis of tissue samples from the 1979 Sverdlovsk anthrax victims: the presence of multiple Bacillus anthracis strains in different victims. Proc Natl Acad Sci USA 95: 1224-9.

9. Jackson PJ, EA Walthers, AS Kalif, KL Richmond, DM Adair et al (1997) Characterization of the variable-number tandem repeats in vrrA from different Bacillus anthracis isolates. Appl Environ Microbiol 63: 1400-5.

10. Keim P, L B Price, AM Klevytska, K L Smith, J M Schupp et al (2000) Multiplelocus variable-number tandem repeat analysis reveals genetic relationships within Bacillus anthracis. J Bacteriol 182: 2928-36.

11. Fasanella A, S Losito, T Trotta, R. Adone, S Massa et al (2001) Detection of anthrax vaccine virulence factors by polymerase chain reaction. Vaccine 19: 4214-8.

12. Keim P, A M Klevytska, L B Price, J M Schupp, G Zinser, et al (1999) Molecular diversity in Bacillus anthracis. J Appl Microbiol 87: 215-7.

13. Price LB, M Hugh-Jones, PJ Jackson, and P Keim (1999) Genetic diversity in the protective antigen gene of Bacillus anthracis. J Bacteriol 181: 2358-62.

14. Ramisse V, G Patra, H Garrigue, J L Guesdon, and M Mock (1996) Identification and characterization of Bacillus anthracis by multiplex PCR analysis of sequences on plasmids pXO1 and pXO2 and chromosomal DNA. FEMS Microbiol Lett 145: 9-16.

15. Patra G, P Sylvestre, V Ramisse, J Therasse, and J L Guesdon (1996) Isolation of a specific chromosomic DNA sequence of Bacillus anthracis and its possible use in diagnosis. FEMS Immunol Med Microbiol 15: 223-31.

\section{Citation:}

Shulman LM, David D, Mendelson E, Elad D (2019) How safe is your DNA extract? Integr J Vet Biosci Volume 3(1): 1-4. 